\title{
Domain Balanced Sampling and Iterative Search for Product Identification
}

\author{
Litong Gong ${ }^{1,3}$, Sheng Tang ${ }^{1,2,}$, Juan Cao ${ }^{1}$ \\ ${ }^{1}$ Key Laboratory of Intelligent Information Processing, Institute of Computing Technology, Chinese Academy of \\ Sciences, Beijing, China. \\ ${ }^{2}$ Hangzhou Institude for Advanced Study, University of Chinese Academy of Sciences, Hangzhou, China. \\ ${ }^{3}$ University of Chinese Academy of Sciences, Beijing, China. \\ \{gonglitong19s,ts,caojuan\}@ict.ac.cn
}

\begin{abstract}
This paper introduces our solution to the 1st Workshop on Multimodal Product Identification in Livestreaming and Watch and Buy Challenge, a real-world task in a live stream scene and is challenging due to factors such as lighting, occlusion, and cross-domain. We model this task as the object detection and image retrieval problem. In the whole pipeline, we mainly focus on the problem of crossdomain retrieval and propose a domain-balanced sampling method, which enhances the robustness of multi-domain retrieval. Besides, to eliminate the influence of irrelevant clothing, we propose an iterative cross-search strategy, which greatly improves the accuracy of matching. In addition, we also experiment with the exploitation of text information, including multimodal product classification and multimodal intent recognition. With the aforementioned method, we achieved an F1 score of $69.2 \%$ and finally achieve first place in the competition.
\end{abstract}

\section{CCS CONCEPTS}

- Information systems $\rightarrow$ Multimedia and multimodal retrieval.

\section{KEYWORDS}

Fine-grained retrieval, Mutilmodal product recognition

\section{ACM Reference Format:}

Litong Gong ${ }^{1,3}$, Sheng Tang ${ }^{1,2,}$, Juan $\mathrm{Cao}^{1}$. 2021. Domain Balanced Sampling and Iterative Search for Product Identification. In Proceedings of the 1st Workshop on Multimodal Product Identification in Livestreaming and WAB Challenge (WAB '21), Oct. 24, 2021, Virtual Event, China. ACM, New York, NY, USA, 8 pages. https://doi.org/10.1145/3475956.3484483

\footnotetext{
* Corresponding author.

This work was supported by the National Key Research and Development Program of China (2017YFC0820605), and the National Natural Science Foundation of China (U1703261, 61871004), and the Project of Chinese Academy of Sciences (E141020).
}

Permission to make digital or hard copies of all or part of this work for personal or classroom use is granted without fee provided that copies are not made or distributed for profit or commercial advantage and that copies bear this notice and the full citation on the first page. Copyrights for components of this work owned by others than ACM must be honored. Abstracting with credit is permitted. To copy otherwise, or republish, to post on servers or to redistribute to lists, requires prior specific permission and/or a fee. Request permissions from permissions@acm.org.

WAB '21, October 24, 2021, Virtual Event, China.

(c) 2021 Association for Computing Machinery.

ACM ISBN 978-1-4503-8677-7/21/10 . \$ \$15.00

https://doi.org/10.1145/3475956.3484483

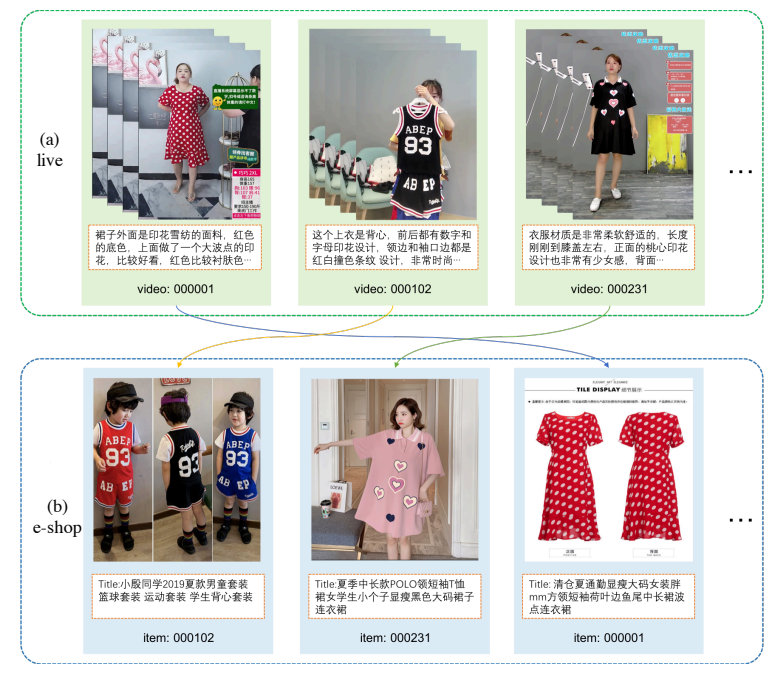

Figure 1: Visualization of the WAB product recognition task. (a) The video clips of the clothing in a live scene. (b) The information about the clothing in the e-shop. The WAB product recognition task is to match the products in (a) with those in (b) and give the category labels of the products. We can use visual information, product title, and video speechto-text information to assist in product recognition.

\section{INTRODUCTION}

Watch and Buy(WAB) Multimodal Product Identification Challenge is a workshop held at ACM MM2021, which focuses on the problem of multimodal product recognition in live streaming. This paper is our solution on this track.

With the rise of live e-commerce in recent years, consumers can watch live streams and purchase products on various live streaming platforms. In a live broadcast, the host introduces hundreds of products, and the viewer has to find specific products from a pregiven product library, which is inconvenient for the consumer. $\mathrm{WAB}$ challenge focuses on the problem of matching the products displayed in the live streaming with the products in the e-shop and is dedicated to automatically and accurately recommending the product being explained to the consumer. The task is described as shown in the Figure 1.

There are many challenges in the WAB multimodal product recognition task. The first challenge is that recognizing the same 
product requires a fine-grained product retrieval model to be robust enough due to differences in color, distortion, occlusion, and display form of the product. To address this, we use the autoaugmentation $[7,58]$ method imposed to object detection and image retrieval for data enhancement respectively, which can greatly improve the robustness of the model. In addition, we employ the backbone with the IBN [33] structure for training the image retrieval model, which can also increase the generalization ability of the model in new scenes.

The second challenge is the cross-domain differences between the product images. Since the WAB product recognition task is to match the clothing products in the live stream scene with the e-shop images, and there are many differences in lighting and display forms between the two. How to cover both domains when extracting product features is a very important topic. To address this, we propose the domain balanced $\mathrm{P}-\mathrm{K}$ (DB-PK) sampling strategy [17] of the image retrieval model, which ensures that samples from multiple domains need to be covered within the same batch during the training process, instead of random sampling.

The third challenge is the problem of imbalance in clothing categories. When recognizing clothing categories, it is a concern to accurately identify the categories with fewer samples. To address this, we employ seesaw loss [49] to improve the accuracy of the object detection model to classify on the tail category. We also try to use multimodal information to model the clothing categories by using multiple product images from e-shop with clothing title information.

The fourth challenge is the interference of irrelevant costumes. In the live streaming scenario, the object detection model detects clothes that exist outside of the clothes being displayed by the host, and if all the detection results are used for similarity calculation, the noise would be introduced, leading to matching failure. To address this, we propose an iterative cross-search(ICS) strategy, which is a post-processing strategy that eliminates as many irrelevant clothes as possible and retrieves only the clothes being displayed by the host, which greatly improves the matching accuracy.

In summary, our contributions are summarized as follows.

- We develop a comprehensive pipeline for the WAB product recognition task and conduct detailed experiments based on recent years of research, especially on fine-grained retrieval

- We propose a DB-PK sampling method for the cross-domain retrieval problem, which maximally reduces the variability among multiple domains and improves the robustness of the retrieval model.

- We propose an iterative cross-search post-processing strategy for the problem of irrelevant dresses in the WAB product recognition problem, which accurately identifies the anchor intent and greatly reduces the impact of irrelevant clothing on retrieval matching.

- We achieve $69.2 \%$ in F1 score and win the first place in the challenge.

\section{RELATED WORK}

\subsection{Object Detection}

General Object Detection. The current object detection methods are divided into anchor-based methods [12, 23, 25, 38, 39, 43] and anchor-free methods [11, 22, 45, 55, 56]. The anchor-based methods include one-stage methods and two-stage methods. The one-stage detector $[25,27,38]$ generates the detection results directly from the multi-scale feature maps without the process of pre-selected region generation. The two-stage detector $[4,39]$ first generates pre-selected boxes through the Region Proposal Network (RPN) network, and then employs the detection head to finetune the candidate boxes and generate the detection results. The two-stage network tends to achieve higher performance in terms of accuracy but at a slightly slower speed.

Long Tail Recognition. The long tail recognition task [14, 15, $28,50,52]$ is emerging as a hot topic due to its common existence in the real world. A general approach is to use re-weighting $[5,8,20]$ for the loss, and another common approach $[19,30]$ is to re-balance the distribution of the instance numbers in each class. Recently, many works decouple the training process using a multi-stage training procedure. Besides, some works modify the classifier, such as the balanced group softmax loss [24]. The seesaw loss [49] is proposed to dynamically suppress the excessive negative sample gradients on the tail category and to complement the penalty for misclassified samples. The seesaw loss significantly improves the classification accuracy of the tail category.

\subsection{Image retrieval}

Feature Extractor. The methods for image retrieval [1, 34, 53] can be divided into two categories, which are local feature-based methods [3, 32, 44] and global feature-based methods [13, 35, 36, 46]. The local feature-based methods can aggregate manual features. With the rise of deep learning, convolutional neural networks provide a global description approach. With the global description approach, only one forward pass is required to obtain the final representation. Moreover, convolutional neural networks have undergone several changes in pooling methods, which comes in the form of max-pooling [46], SPoC[2], CroW [21], R-MAC [46] and GeM [36]. Among them, GeM has become the most dominant pooling method in image retrieval nowadays due to its adaptive weighted aggregation.

Pair-Based DML. The pair-based deep metrics learning(DML) [6, 40-42, 51] optimizes the distribution of feature space by computing the similarity of sample pair levels, which has gradually become one of the most commonly used loss functions in image retrieval tasks. The contrast loss [6] can learn features with distinguishing power through siamese networks, which also drives the whole field of deep metric learning and image retrieval. The triplet loss [18, 40] requires the similarity between positive sample pairs to be greater than that between negative sample pairs by a given margin. The proposed PK sampling [17] makes it possible to extract hard positive and hard negative pairs online, which greatly improves the effectiveness of the triplet loss. In recent years, the pair-based DML methods have emerged, such as N-pair loss [41], Multi-Similarity (MS) loss [51], which can be adaptively weighted for the given pairs. The circle loss [42] is proposed to unify the pair-based loss with the softmax-based loss, which achieves promising results in the fields of face recognition, person re-identification, and metric learning. 


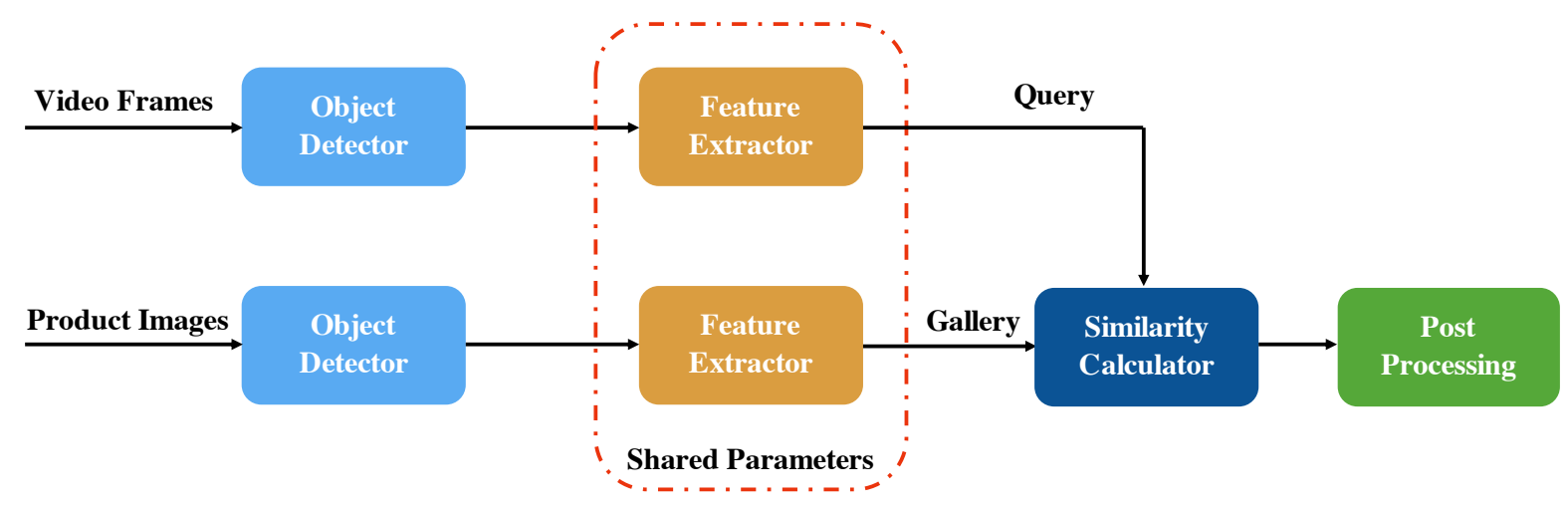

Figure 2: Illustration of the whole framework of our method. Our method contains a total of two object detectors, a feature extractor, a similarity calculator, and a post-processing module. The clothing images in the live scene are used as the query set, while the images of the product library are used as the gallery set.
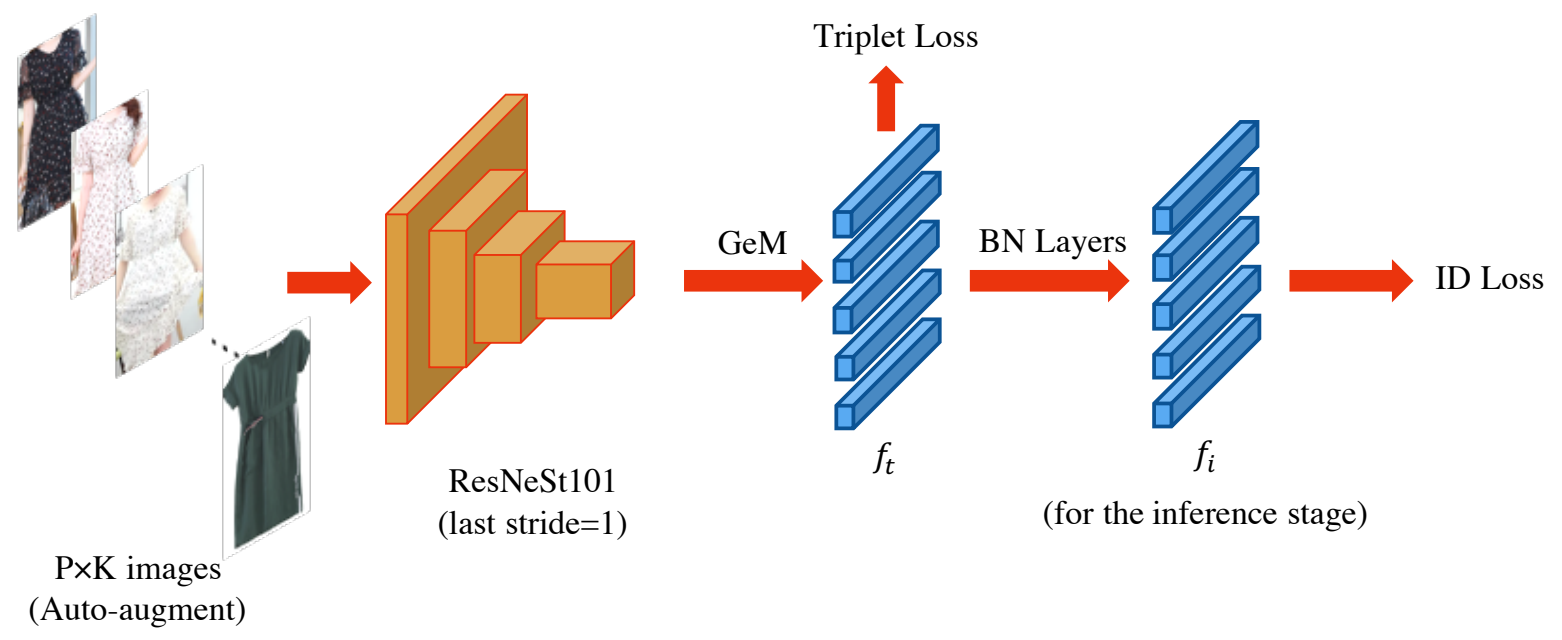

(for the inference stage)

Figure 3: Illustration of the feature extraction model. After domain balanced P-K sampling, it is input to the ResNeSt101 [54] and finally pooled by GeM [36] to get the feature $f_{t}$, which is used for the training of triplet loss. $f_{t}$ is passed through the BN layer to get $f_{i}$, which is used for the training of identity loss. In the testing phase, $f_{i}$ is used for the final representations.

\section{THE PROPOSED METHOD}

\subsection{Overall architecture}

We design a two-stage pipeline to solve the WAB product recognition problem. The whole framework is shown in Figure 2. In the first phase, we employ an object detector to detect the apparel of video frames and product images from live scenes, and the detection results contain 23 categories. In the second stage, we input the results of object detection into the image retrieval model to extract the features of each clothing item. Finally, we perform a nearest neighbor search for the clothing from the live video, and the clothing from the e-shop images, then after merging and postprocessing the results, we obtain the item id corresponding to each live video.

Next, we describe the details of each part in further detail.

\subsection{Object Detection}

In the $\mathrm{WAB}$ product recognition task, we find that the localization performance of the objects is of secondary importance, while the classification of the clothing category is more important. We analyze that the location information of the apparel only provides the location information to the retrieval model, and the robustness of the retrieval model makes the localization of the object not so important. On the other hand, since the metric calculates the F1 score by category, the labeling of clothing is very important, especially on categories with few samples.

We employ Cascade R-CNN [4] as the solution for object detection and ResNet50 [16] as the backbone. To address the different shapes of the clothing, we employ DCN-V2 [57] instead of the original convolution operator. In addition, we increase the loss weight of the classification by double to improve the recognition accuracy 


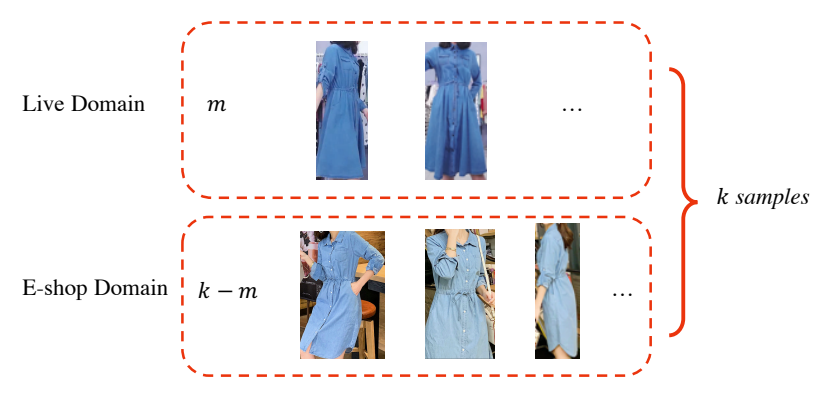

Figure 4: Illustration of the domain balanced P-K sampler.

of the clothing category. We also try to employ seesaw loss [49] to further improve the accuracy in categories with few samples, which contributes to the improvement of the F1 score.

\subsection{Feature Extractor}

In this section, we perform a fine-grained retrieval of the clothing. The whole model framework is shown in Figure 3.

Data Processing and Augmentation. We first filter out the clothing without matching pairs for the training samples. During the training process, we resize all images to the same size, while using auto-augmentation [7] as our data augmentation, all strategies are searched on ImageNet [9] to achieve this, which can be well transferred to the WAB product recognition task.

Network Structure. To identify fine-grained clothing, we try the work [29] in the pedestrian re-identification task as our baseline model. The structure of the whole image retrieval model is shown in Figure 3. On the basis of baseline, we use ResNet101 [16] and ResNeSt101 [54] pre-trained on ImageNet [9] as backbone. In addition, the IBN structure [33] is used to enhance the ability of the model to adapt to different domains. Moreover, we set the step size of the last layer of the backbone to 1 to enhance the size of the feature map and extract more fine-grained features. Furthermore, we employ generalized mean-pooling (GeM) [36] to obtain the final feature vector, which shows a great advantage in the clothing retrieval task.

Loss Function. For the fine-grained retrieval task, we employ both the ID loss and triplet loss [40] to train the model. For ID loss, we employ circle loss [42], which is better than Arcface [10] and cross-entropy loss. And for triplet loss, it is combined with modified PK sampling [17] to better mine harder samples.

Domain Balanced Sampler. For the WAB product recognition task, the retrieved query set, and gallery set are from two different domains, live scene and e-shop images respectively, and there are serious domain differences. Therefore, we propose the domain balanced PK(DB-PK) sampling strategy, for each batch, sampling $k$ categories, and sampling $p$ samples per category, we need to do some restrictions on $p$ samples. As shown in the Figure 4, we need to ensure that there are $m$ samples from the live scene, and the remaining $p-m$ samples from the commodity graph, which can ensure that in each batch, the samples of both domains are covered.

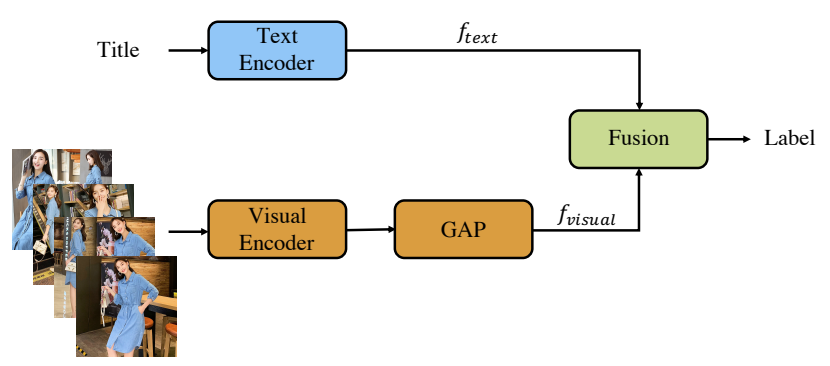

Figure 5: Illustration of the multimodal product classifier. The text encoder and visual encoder use transformer [47] and ResNet50 [16], respectively, and the fusion module uses transformer for classification through two FC layers.

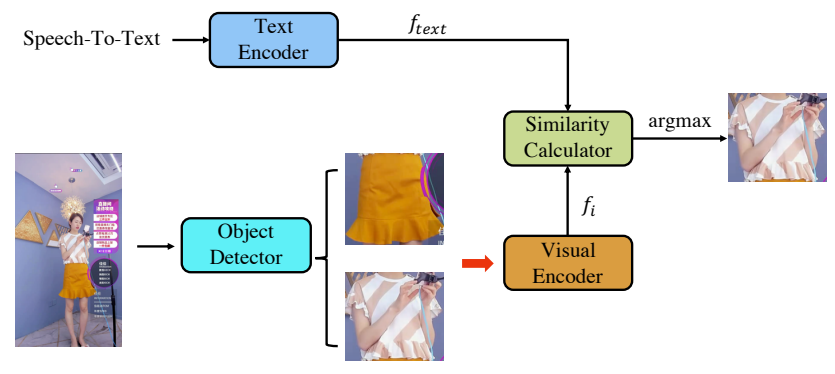

Figure 6: Illustration of the multimodal intent recognition. The training process of this module is similar to CLIP [37]. In the testing phase, for each frame, the clothing image with the highest similarity to the text feature $f_{\text {text }}$ is always selected.

\subsection{Multimodal Modeling}

In this competition, besides visual information, speech-to-text and product title information are also provided. For this, we also try two ideas.

Multimodal Product Classification. Because of the serious sample imbalance in the clothing category, and the differences between clothing categories are not clearly distinguishable, such as vest top and sling top. Whereas in the product title, there are frequently special keywords that can indicate the category of the product. Therefore, we consider the use of e-shop images together with product titles for product category classification. The detailed structure is shown in Figure 5. We input the title and e-shop image together into the model, extract text features and image features respectively, and then perform multimodal fusion by transformer for final classification.

Multimodal Intent Recognition. Under live streaming, the target detector detects all the clothing of the current video frame, but among them, only one clothing is being talked about by the host, therefore, there are a large number of irrelevant clothing in this situation. We try to use speech-to-text messages for filtering irrelevant clothing because the speech message usually describes the clothing that the host is talking about. Therefore, we design the pipeline as in Figure 6, the text description is encoded to get the text features, and each clothing image is encoded to get the 
(a)

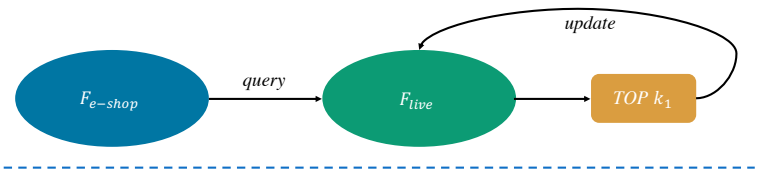

(b)

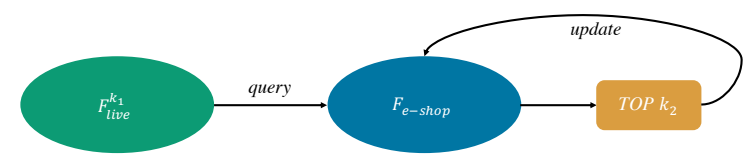

Figure 7: Illustration of the iterative cross-search. (a). Using images in the e-shop domain to retrieve images in the live domain and keeping $k_{1}$ the most similar images. (b). Using images in the live domain to retrieve images in the e-shop domain and keeping $k_{2}$ the most similar images. The iterative cross-search repeatedly performs (a) and (b) sequentially, and finally obtains the set of images with irrelevant clothing removed.

visual features, and the two are calculated for similarity, finally, we choose the object with the highest similarity to the corresponding text as the relevant clothing for the current frame. Theoretically, this method can better identify the intention of the host and minimize the interference of irrelevant clothing.

\subsection{Post Processing}

The post-processing can significantly improve the accuracy of product recognition in the inference phase. In this section, we introduce several post-processing techniques of our approach.

Augmentation Test. For each test image, we employ the original image and the flipped image as input in the target detector to obtain two groups of results, respectively and perform NMS [31] processing on them to obtain the final costume detection results. Similarly, for the image retrieval model, we use the original image and the level-flipped image as inputs to get two groups of features, which are retrieved separately and the results are fused.

Iterative Cross-Search. To remove the irrelevant clothing detected by the object detector, we propose an iterative cross-search strategy to solve this problem. Specifically, we define the set of clothing frame results for the live scene as $B_{\text {live }}=\left\{b_{0}, \ldots . b_{N_{1}}\right\}$, and the extracted features are $F_{\text {live }}=\left\{f_{0} \ldots f_{N_{1}}\right\}$ The detection result of e-shop images is $B_{\text {item }}=\left\{b_{0}, \ldots . b_{N_{2}}\right\}$, and the extracted features are $F_{\text {item }}=\left\{f_{0} \ldots . f_{N_{2}}\right\}$, where $N_{1}$ and $N_{2}$ denote the size of the set. First, we retrieve $F_{\text {live }}$ using $F_{\text {item }}$ and select the top $K_{1}$ results to keep, we consider most of the other results as irrelevant clothes and discard them to obtain $F_{\text {live }}^{k_{1}}$. Then, we use $F_{\text {live }}^{k_{1}}$ to retrieve $F_{\text {item }}$ and select the top $K_{2}$ results to keep, we consider most of the other results are irrelevant clothes and discard them to get $F_{\text {item }}^{k_{2}}$. After repeating the above process for iterative cross-search, finally, we get $F_{\text {live }}^{k_{i}}$ and $F_{\text {item }}^{k_{i+1}}$ which is the set of cleaned clothing features. In our experiments, $i=5$ filters out $70 \%$ of irrelevant clothes, while $10 \%$ of relevant clothes with poor image quality are filtered out. The iterative cross-search strategy can significantly improve the matching accuracy.
Label Prediction. We use the results of the object detector on the product image to generate the final category labels. Specifically, we compute histograms of category scores for all successfully matched clothing images. The category with the highest score becomes the final category for the product.

Variance Results Generation. Due to the presence of suitlike clothing in the dataset. Therefore, the differentiated results allow the returned result list to cover as many suits as possible. Specifically, after we return the result with the highest confidence in the detection result, the second-ranked result is guaranteed to be in a different category with a high enough confidence level. This returns a higher probability of suits.

\section{EXPERIMENTS}

\subsection{Datasets and Evaluation Protocol}

Watch and Buy (WAB) is a large-scale multimodal clothing dataset containing 70,000 video clips and clothing item matching pairs. For each video clip, 10 keyframes are annotated at the instance level, along with the corresponding speech recognition text. For each item, the image is annotated and the text of the item's title is provided. In total, 1,042,178 images were annotated with $1,654,780$ bounding boxes.

The training dataset consists of 50,000 matched pairs containing video clips extracted from a live stream of Taobao Live and product store images. Annotations include product categories, bounding boxes, viewpoint types, display types and instance ids, title descriptions, and speech recognition text. An example is shown below.

Evaluation Protocol. In our experiments, we use macro F1 scores for evaluation. Since different product categories have different numbers of test instances, F1 scores are calculated for each category individually and the final score is the average F1 score over all categories. The true positive instance should satisfy the following two conditions: 1 ). The item id is the same as the ground truth, 2). IoU between retrieved item and one of the ground truth boxes with the same label is over the thresh(we set the threshold as 0.5$)$.

\subsection{Implementation Details}

Object Detection. We use Cascade R-CNN [4] to model the clothing of the live scenes and the costumes of the merchandise images separately. We employ multi-scale for training, with input sizes of $400-800$, and auto-augmentation for data augmentation. In addition, the classification loss is twice the localization loss to train the model. We use an SGD optimizer and train 12 epochs. In the testing phase, the input size of the image is 600 , and 0.7 is chosen as the threshold for clothing detection. All the pre-training is used is obtained by training on the COCO dataset [26].

Image Retrieval. We resize the input image to $256 \times 256$ and do horizontal flipping with auto-augmentation on the dataset. In DBPK sampling, we set $P=3, K=40, m=1$. We employ ResNeST101 and ResNet101-IBN-A as the backbone. in the training phase, we used circle loss with scale $=128$, margin $=0.3$, and triplet loss with margin $=0.3$, both with a loss weight of $1: 9$. Furthermore, we train the Adam optimizer with an initial learning rate of $7 \mathrm{e}-4$ for 100 epochs, and the learning rate is reduced to 0.1 at 35 epochs and 
70 epochs, respectively. In addition, the warm-up strategy is also employed for the first 10 epochs.

\subsection{Ablation Study}

Table 1: Comparison of different object detectors. All experiments are tested in the validation set. The mAP is the metric for object detection. The F1 score is the metric for the entire pipeline, and in this experiment, the image retrieval model uses our baseline(ResNet50 + CE + Triplet Loss).

\begin{tabular}{lcc}
\hline Methods & $\mathrm{mAP}(\%)$ & $\mathrm{F} 1$ score $(\%)$ \\
\hline Faster R-CNN & 68.3 & 53.7 \\
\hline Cascade R-CNN & 70.7 & 55.9 \\
+ DCN V2 & 72.0 & 56.2 \\
+ Auto Augment & 72.7 & 57.3 \\
+ Seesaw Loss & 71.8 & 57.6 \\
\hline
\end{tabular}

Comparison of different object detectors. We try the impact of different object detection structures on the validation set, and we use Faster-R-CNN and Cascade R-CNN for object detection, respectively. It is shown that although Cascade R-CNN works well for object detection, it has limited improvement on the final F1 score. This is because object detection is only an upstream task, and it is the feature extractor that plays a key role. In addition, we conduct ablation experiments on data augmentation and deformable convolution for object detection, etc., as shown in Table 1.

Table 2: Comparison of the different backbone of image retrieval models. R-1 indicates the matching accuracy of TOP 1. The F1 score is the metric of the competition. All experiments are tested in the validation set. Ensemble indicates model fusion using the results of ResNet101-IBN-A and ResNeSt101.

\begin{tabular}{lcc}
\hline Methods & R-1(\%) & F1 score(\%) \\
\hline ResNet-50 & 79.8 & 57.6 \\
ResNet-50-IBN-A & 81.5 & 57.8 \\
ResNet-101-IBN-A & 83.6 & 59.9 \\
ResNeSt-101 & 84.4 & 60.5 \\
\hline Ensemble & 85.5 & 61.2 \\
\hline
\end{tabular}

Comparison of different backbone for retrieval model. On the validation set, we compare the effect of different backbones on the results. For fair comparison, we use a $256 \times 256$ image as input. As shown in Table 2, a larger convolutional neural network can enhance the representation of features. The use of IBN can further improve the generalization ability of the model. We finally choose ResNet101-IBN-A and ResNeSt101 as feature extractors for model fusion.

Comparison of different loss functions for the retrieval model. In the feature extraction model training process, the appropriate loss function can make the model converge more quickly and keep the features closer together for the same item and further
Table 3: Comparison of different loss functions for the retrieval model. The backbone of all experiments is ResNeSt101.

\begin{tabular}{lcc}
\hline Loss & R-1(\%) & F1 score(\%) \\
\hline CE + Triplet Loss & 84.4 & 60.5 \\
AM Sofrmax [48] + Triplet Loss & 85.5 & 62.1 \\
Arcface [10] + Triplet Loss & 85.9 & 62.1 \\
Circle Loss [42] + Triplet Loss & 87.0 & 62.7 \\
\hline
\end{tabular}

apart for different clothing. In Table 3, we perform ablation experiments on the commonly used loss functions, and finally, we choose to circle loss as id-based loss and triplet loss as pair-based loss.

Table 4: Ablation experiments with multimodal modeling. The strong baseline means the model with ResNeSt-101 and circle Loss.

\begin{tabular}{lc}
\hline Methods & F1 score $(\%)$ \\
\hline Strong Baseline(SB) & 62.7 \\
\hline SB + Multimodal Product Classification & 62.5 \\
SB + Multimodal Intent Recognition & 62.0 \\
\hline
\end{tabular}

Ablation experiments with multimodal modeling. As shown in Table 4, we explore the impact of the two multimodal modeling approaches on the results in the validation set. The use of multimodal product recognition is almost consistent compared to baseline. While using multimodal intent recognition, the results are not as good as using iterative cross-search. Our conjecture is that the training process of the text encoder is not guided by a large-scale linguistic pre-training model, which makes the text representation not as effective as expected. How to use multimodal information appropriately is well worth exploring in the future. In our final version, multimodal information modeling is not included.

Table 5: Ablation experiments of different parts of the method. The strong baseline means the model with ResNeSt101 and circle Loss.

\begin{tabular}{lcc}
\hline Methods & $\mathrm{R}-1(\%)$ & $\mathrm{F} 1$ score $(\%)$ \\
\hline Strong Baseline & 87.0 & 62.7 \\
\hline + Auto Augmentation & 89.0 & 63.3 \\
+ DB-PK sampler & 90.7 & 64.2 \\
+ Iterative Cross-Search & 93.1 & 65.3 \\
+ Variance Results Generation & 93.1 & 67.2 \\
+ Ensemble & 94.2 & 67.9 \\
\hline
\end{tabular}

Other ablation experiments. We test the effectiveness of different parts of our method. As shown in Table 5, using auto-augmentation has a $2.0 \%$ boost on the matching performance. A further $1.7 \% \mathrm{im}-$ provement can be achieved using our designed DB-PK sampling method. Finally, we can substantially filter out irrelevant costumes 
using iterative cross-search with a $2.4 \%$ improvement over direct result fusion. Further, we generate a list of results with discrepancies, which improves the hit rate of the suit, gaining a $1.9 \%$ gain in F1 score.

Table 6: Results of the WAB challenge in the final stage. All models are trained by using all data, and the competition organizers reproduce the training models and testing results.

\begin{tabular}{lcc}
\hline Rank & Team Name & F1 score $(\%)$ \\
\hline $\mathbf{1}$ & mcg(ours) & $\mathbf{6 9 . 2}$ \\
\hline 2 & code written & 64.9 \\
3 & 10 billion subsidies & 62.3 \\
4 & THU & 50.2 \\
5 & Crow in a plane & 49.1 \\
\hline
\end{tabular}

\subsection{Competitive results}

Our team finally achieves the F1 score of $69.2 \%$ in the final stage. As the Table 6 shows, we win the competition with a $4.3 \%$ advantage in F1 score.

\section{CONCLUSIONS}

In this paper, we summarize the technical solution of the WAB multimodal product recognition challenge. We focus on solving the cross-domain retrieval problem and the interference problem of irrelevant clothing, which substantially improves the accuracy of clothing matching. Besides, we also explore the utilization of multimodal information, and we believe that the assistance of multimodal information will further improve the performance of WAB in the future. We finally achieved an F1 score of $69.2 \%$ and won the competition.

\section{REFERENCES}

[1] Relja Arandjelović and Andrew Zisserman. 2012. Three things everyone should know to improve object retrieval. In 2012 IEEE Conference on Computer Vision and Pattern Recognition. IEEE, 2911-2918.

[2] Artem Babenko and Victor Lempitsky. 2015. Aggregating deep convolutional features for image retrieval. arXiv preprint arXiv:1510.07493 (2015).

[3] Herbert Bay, Tinne Tuytelaars, and Luc Van Gool. 2006. Surf: Speeded up robust features. In European conference on computer vision. Springer, 404-417.

[4] Zhaowei Cai and Nuno Vasconcelos. 2018. Cascade r-cnn: Delving into high quality object detection. In Proceedings of the IEEE conference on computer vision and pattern recognition. 6154-6162.

[5] Kaidi Cao, Colin Wei, Adrien Gaidon, Nikos Arechiga, and Tengyu Ma. 2019 Learning imbalanced datasets with label-distribution-aware margin loss. arXiv preprint arXiv:1906.07413 (2019).

[6] Sumit Chopra, Raia Hadsell, and Yann LeCun. 2005. Learning a similarity metric discriminatively, with application to face verification. In 2005 IEEE Computer Society Conference on Computer Vision and Pattern Recognition (CVPR'05), Vol. 1. IEEE, 539-546.

[7] Ekin D Cubuk, Barret Zoph, Dandelion Mane, Vijay Vasudevan, and Quoc V Le. 2019. Autoaugment: Learning augmentation strategies from data. In Proceedings of the IEEE/CVF Conference on Computer Vision and Pattern Recognition. 113-123.

[8] Yin Cui, Menglin Jia, Tsung-Yi Lin, Yang Song, and Serge Belongie. 2019. Classbalanced loss based on effective number of samples. In Proceedings of the IEEE/CVF conference on computer vision and pattern recognition. 9268-9277.

[9] Jia Deng, Wei Dong, Richard Socher, Li-Jia Li, Kai Li, and Li Fei-Fei. 2009. Imagenet A large-scale hierarchical image database. In 2009 IEEE conference on computer vision and pattern recognition. Ieee, 248-255.

[10] Jiankang Deng, Jia Guo, Niannan Xue, and Stefanos Zafeiriou. 2019. Arcface: Additive angular margin loss for deep face recognition. In Proceedings of the IEEE/CVF Conference on Computer Vision and Pattern Recognition. 4690-4699.
[11] Kaiwen Duan, Song Bai, Lingxi Xie, Honggang Qi, Qingming Huang, and Qi Tian. 2019. Centernet: Keypoint triplets for object detection. In Proceedings of the IEEE/CVF International Conference on Computer Vision. 6569-6578.

[12] Ross Girshick, Jeff Donahue, Trevor Darrell, and Jitendra Malik. 2015. Regionbased convolutional networks for accurate object detection and segmentation. IEEE transactions on pattern analysis and machine intelligence 38, 1 (2015), 142158.

[13] Albert Gordo, Jon Almazán, Jerome Revaud, and Diane Larlus. 2016. Deep image retrieval: Learning global representations for image search. In European conference on computer vision. Springer, 241-257.

[14] Agrim Gupta, Piotr Dollar, and Ross Girshick. 2019. LVIS: A dataset for large vocabulary instance segmentation. In Proceedings of the IEEE/CVF Conference on Computer Vision and Pattern Recognition. 5356-5364.

[15] Haibo He and Edwardo A Garcia. 2009. Learning from imbalanced data. IEEE Transactions on knowledge and data engineering 21, 9 (2009), 1263-1284.

[16] Kaiming He, Xiangyu Zhang, Shaoqing Ren, and Jian Sun. 2016. Deep residual learning for image recognition. In Proceedings of the IEEE conference on computer vision and pattern recognition. 770-778.

[17] Alexander Hermans, Lucas Beyer, and Bastian Leibe. 2017. In defense of the triplet loss for person re-identification. arXiv preprint arXiv:1703.07737 (2017).

[18] Elad Hoffer and Nir Ailon. 2015. Deep metric learning using triplet network. In International workshop on similarity-based pattern recognition. Springer, 84-92.

[19] Xinting Hu, Yi Jiang, Kaihua Tang, Jingyuan Chen, Chunyan Miao, and Hanwang Zhang. 2020. Learning to segment the tail. In Proceedings of the IEEE/CVF Conference on Computer Vision and Pattern Recognition. 14045-14054.

[20] Chen Huang, Yining Li, Chen Change Loy, and Xiaoou Tang. 2019. Deep imbalanced learning for face recognition and attribute prediction. IEEE transactions on pattern analysis and machine intelligence 42, 11 (2019), 2781-2794.

[21] Yannis Kalantidis, Clayton Mellina, and Simon Osindero. 2016. Cross-dimensional weighting for aggregated deep convolutional features. In European conference on computer vision. Springer, 685-701.

[22] Hei Law and Jia Deng. 2018. Cornernet: Detecting objects as paired keypoints. In Proceedings of the European conference on computer vision (ECCV). 734-750.

[23] Yu Li, Sheng Tang, Min Lin, Yongdong Zhang, Jintao Li, and Shuicheng Yan. 2018. Implicit negative sub-categorization and sink diversion for object detection. IEEE Transactions on Image Processing 27, 4 (2018), 1561-1574.

[24] Yu Li, Tao Wang, Bingyi Kang, Sheng Tang, Chunfeng Wang, Jintao Li, and Jiashi Feng. 2020. Overcoming classifier imbalance for long-tail object detection with balanced group softmax. In Proceedings of the IEEE/CVF conference on computer vision and pattern recognition. 10991-11000.

[25] Tsung-Yi Lin, Priya Goyal, Ross Girshick, Kaiming He, and Piotr Dollár. 2017. Focal loss for dense object detection. In Proceedings of the IEEE international conference on computer vision. 2980-2988.

[26] Tsung-Yi Lin, Michael Maire, Serge Belongie, James Hays, Pietro Perona, Deva Ramanan, Piotr Dollár, and C Lawrence Zitnick. 2014. Microsoft coco: Common objects in context. In European conference on computer vision. Springer, 740-755.

[27] Wei Liu, Dragomir Anguelov, Dumitru Erhan, Christian Szegedy, Scott Reed, Cheng-Yang Fu, and Alexander C Berg. 2016. Ssd: Single shot multibox detector. In European conference on computer vision. Springer, 21-37.

[28] Ziwei Liu, Zhongqi Miao, Xiaohang Zhan, Jiayun Wang, Boqing Gong, and Stella X Yu. 2019. Large-scale long-tailed recognition in an open world. In Proceedings of the IEEE/CVF Conference on Computer Vision and Pattern Recognition. 2537-2546.

[29] Hao Luo, Youzhi Gu, Xingyu Liao, Shenqi Lai, and Wei Jiang. 2019. Bag of tricks and a strong baseline for deep person re-identification. In Proceedings of the IEEE/CVF Conference on Computer Vision and Pattern Recognition Workshops. 0-0.

[30] Dhruv Mahajan, Ross Girshick, Vignesh Ramanathan, Kaiming He, Manohar Paluri, Yixuan Li, Ashwin Bharambe, and Laurens Van Der Maaten. 2018. Exploring the limits of weakly supervised pretraining. In Proceedings of the European conference on computer vision (ECCV). 181-196.

[31] Alexander Neubeck and Luc Van Gool. 2006. Efficient non-maximum suppression. In 18th International Conference on Pattern Recognition (ICPR'06), Vol. 3. IEEE, 850-855.

[32] Hyeonwoo Noh, Andre Araujo, Jack Sim, Tobias Weyand, and Bohyung Han. 2017. Large-scale image retrieval with attentive deep local features. In Proceedings of the IEEE international conference on computer vision. 3456-3465.

[33] Xingang Pan, Ping Luo, Jianping Shi, and Xiaoou Tang. 2018. Two at once: Enhancing learning and generalization capacities via ibn-net. In Proceedings of the European Conference on Computer Vision (ECCV). 464-479.

[34] Filip Radenović, Ahmet Iscen, Giorgos Tolias, Yannis Avrithis, and Ondřej Chum. 2018. Revisiting oxford and paris: Large-scale image retrieval benchmarking. In Proceedings of the IEEE Conference on Computer Vision and Pattern Recognition. 5706-5715.

[35] Filip Radenović, Giorgos Tolias, and Ondřej Chum. 2016. CNN image retrieval learns from BoW: Unsupervised fine-tuning with hard examples. In European conference on computer vision. Springer, 3-20.

[36] Filip Radenović, Giorgos Tolias, and Ondřej Chum. 2018. Fine-tuning CNN image retrieval with no human annotation. IEEE transactions on pattern analysis and 
machine intelligence 41, 7 (2018), 1655-1668.

[37] Alec Radford, Jong Wook Kim, Chris Hallacy, Aditya Ramesh, Gabriel Goh, Sandhini Agarwal, Girish Sastry, Amanda Askell, Pamela Mishkin, Jack Clark, et al. 2021. Learning transferable visual models from natural language supervision. arXiv preprint arXiv:2103.00020 (2021)

[38] Joseph Redmon and Ali Farhadi. 2018. Yolov3: An incremental improvement. arXiv preprint arXiv:1804.02767 (2018).

[39] Shaoqing Ren, Kaiming He, Ross Girshick, and Jian Sun. 2015. Faster r-cnn Towards real-time object detection with region proposal networks. Advances in neural information processing systems 28 (2015), 91-99.

[40] Florian Schroff, Dmitry Kalenichenko, and James Philbin. 2015. Facenet: A unified embedding for face recognition and clustering. In Proceedings of the IEEE conference on computer vision and pattern recognition. 815-823.

[41] Kihyuk Sohn. 2016. Improved deep metric learning with multi-class n-pair loss objective. In Advances in neural information processing systems. 1857-1865.

[42] Yifan Sun, Changmao Cheng, Yuhan Zhang, Chi Zhang, Liang Zheng, Zhongdao Wang, and Yichen Wei. 2020. Circle loss: A unified perspective of pair similarity optimization. In Proceedings of the IEEE/CVF Conference on Computer Vision and Pattern Recognition. 6398-6407.

[43] Sheng Tang, Yu Li, Lixi Deng, and Yongdong Zhang. 2017. Object localization based on proposal fusion. IEEE Transactions on Multimedia 19, 9 (2017), 21052116.

[44] Marvin Teichmann, Andre Araujo, Menglong Zhu, and Jack Sim. 2019. Detectto-retrieve: Efficient regional aggregation for image search. In Proceedings of the IEEE/CVF Conference on Computer Vision and Pattern Recognition. 5109-5118.

[45] Zhi Tian, Chunhua Shen, Hao Chen, and Tong He. 2019. Fcos: Fully convolutional one-stage object detection. In Proceedings of the IEEE/CVF international conference on computer vision. 9627-9636.

[46] Giorgos Tolias, Ronan Sicre, and Hervé Jégou. 2015. Particular object retrieval with integral max-pooling of $\mathrm{CNN}$ activations. arXiv preprint arXiv:1511.05879 (2015).

[47] Ashish Vaswani, Noam Shazeer, Niki Parmar, Jakob Uszkoreit, Llion Jones, Aidan N Gomez, Łukasz Kaiser, and Illia Polosukhin. 2017. Attention is all you need. In Advances in neural information processing systems. 5998-6008.
[48] Feng Wang, Jian Cheng, Weiyang Liu, and Haijun Liu. 2018. Additive margin softmax for face verification. IEEE Signal Processing Letters 25, 7 (2018), 926-930.

[49] Jiaqi Wang, Wenwei Zhang, Yuhang Zang, Yuhang Cao, Jiangmiao Pang, Tao Gong, Kai Chen, Ziwei Liu, Chen Change Loy, and Dahua Lin. 2021. Seesaw loss for long-tailed instance segmentation. In Proceedings of the IEEE/CVF Conference on Computer Vision and Pattern Recognition. 9695-9704.

[50] Tao Wang, Yu Li, Bingyi Kang, Junnan Li, Junhao Liew, Sheng Tang, Steven Hoi, and Jiashi Feng. 2020. The devil is in classification: A simple framework for long-tail instance segmentation. In European Conference on computer vision. Springer, 728-744.

[51] Xun Wang, Xintong Han, Weilin Huang, Dengke Dong, and Matthew R Scott. 2019. Multi-similarity loss with general pair weighting for deep metric learning. In Proceedings of the IEEE/CVF Conference on Computer Vision and Pattern Recognition. 5022-5030.

[52] Tong Wu, Oingqiu Huang, Ziwei Liu, Yu Wang, and Dahua Lin. 2020. Distributionbalanced loss for multi-label classification in long-tailed datasets. In European Conference on Computer Vision. Springer, 162-178.

[53] Hongtao Xie, Yongdong Zhang, Ke Gao, Sheng Tang, Kefu Xu, Li Guo, and Jintao Li. 2013. Robust common visual pattern discovery using graph matching. Fournal of visual communication and image representation 24, 5 (2013), 635-646.

[54] Hang Zhang, Chongruo Wu, Zhongyue Zhang, Yi Zhu, Haibin Lin, Zhi Zhang, Yue Sun, Tong He, Jonas Mueller, R Manmatha, et al. 2020. Resnest: Split-attention networks. arXiv preprint arXiv:2004.08955 (2020).

[55] Xingyi Zhou, Dequan Wang, and Philipp Krähenbühl. 2019. Objects as points. arXiv preprint arXiv:1904.07850 (2019).

[56] Xingyi Zhou, Jiacheng Zhuo, and Philipp Krahenbuhl. 2019. Bottom-up object detection by grouping extreme and center points. In Proceedings of the IEEE/CVF Conference on Computer Vision and Pattern Recognition. 850-859.

[57] Xizhou Zhu, Han Hu, Stephen Lin, and Jifeng Dai. 2019. Deformable convnets v2: More deformable, better results. In Proceedings of the IEEE/CVF Conference on Computer Vision and Pattern Recognition. 9308-9316.

[58] Barret Zoph, Ekin D Cubuk, Golnaz Ghiasi, Tsung-Yi Lin, Jonathon Shlens, and Quoc V Le. 2020. Learning data augmentation strategies for object detection. In European Conference on Computer Vision. Springer, 566-583. 